\title{
SIMULATION OF THE SURFACE OF METAL SHEET WITH TEXTURED POLYMER COATING BY METHOD OF DIFFUSION-LIMITED AGGREGATION
}

\author{
${ }^{1}$ Olga MAKSIMOVA, ${ }^{1}$ Aleksandr BAIDGANOV, ${ }^{2}$ Tatiana PETROVA, ${ }^{2}$ Andrei MAKSIMOV, \\ 'Vladislav EGOROV
}

\author{
${ }^{1}$ Cherepovets State University, Laboratory of Mathematical and Computer Modeling, Cherepovets, Russian \\ Federation,og62@mail.ru \\ ${ }^{2}$ Cherepovets State University, Department of Physics, Cherepovets, Russian Federation, to 87@bk.ru
}

https://doi.org/10.37904/metal.2019.737

\begin{abstract}
The study of the geometric characteristics of the metal sheet surface with textured polymer coatings is justified by their connection with mechanical and optical properties. Studying of the surface of coated samples using optical microscopy revealed that it consists of grooves that are assembled into star-shaped clusters scattered across the entire surface of the sheet. At first glance, it seems that these defects are arranged randomly. However, resultats of the production tests showed that the type of defects depends on conditions of formation of the polymer coating. A Method for the constructing a geometrical feature of the surface relief of the polymer coating is proposed. The surface in our model is represented as an array of the clusters of interconnected fractal objects. The surface formation is simulated of completed by the method of random walks.
\end{abstract}

Keywords: Textured polymer coatings, multifractal analysis, diffusion-limited aggregation, surface structure, granule

\section{INTRODUCTION}

Surface roughness appears as defects in many types of products. However, in the last decade, there has been a need for new materials with additional design functions, for example, textured polymer coatings of sheet metals. The surface roughness affects mechanical and optical properties of metals with polymer coating. However, theoretical study of these properties is possible only under the condition that the geometrical function of the considered defects is known.

The purpose of this work is to develop a model that allows the building 3D function describingthe reliefe of textured polymer coating, depending on the conditions of its formation on the sheet metal.

\section{MULTIFRACTAL MODEL}

There are works [1,2], in which the relief of real rough surfaces of solids were described by fractal functions (Weierstrass, etc.). The surface structure of various samples of sheet rolled metal with polyester textured coatings by the method of optical microscopy (OM) showned that the relief of this surface cannot be described also by analytical functions [3]. Besides, the surfaces simulated in ref. [3] have defects of only symmetric form. Figure 1 shows the surface texture of polyester finishing enamel, obtained by means of the optical microscope with the 56-fold magnification. It is obvious that the defects are anisotropic and have a star-shaped form.

The coating, which has a rough, surface, is created due to the presence of texturizing filler in the paintwork material: polyacryl granules, polyamide, etc. The sizes of the granules vary in the range of 1-10 microns. We simulate the such surface relief using multifractal analysis $[4,5]$. The coating surface is represented as an array of interconnected fractal objects, Therefore, we simulated the such relief on two stages. The first stage of the modeling is the study of the location of the granules. The second stage of the simulation is the study of flocculation of polymer molecules on the granules. 


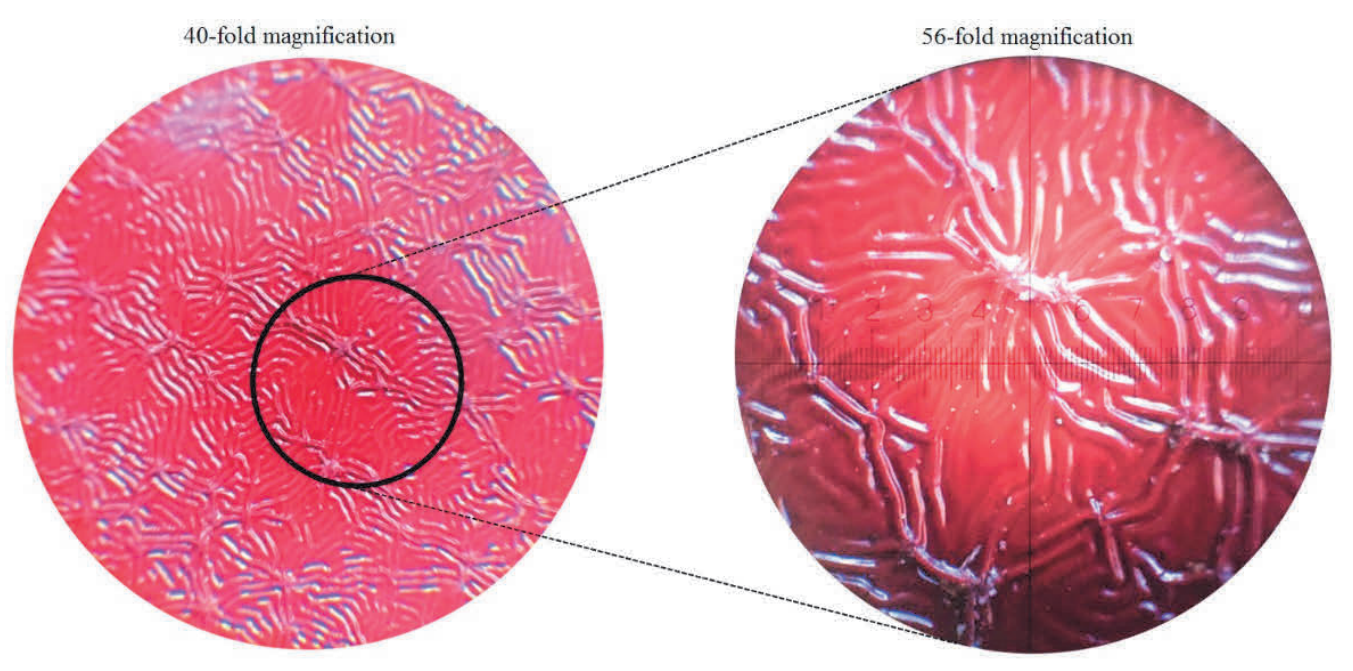

Figure 1 Image of the surface of metal sheet with a polyester textured coating obtained by means an optical microscope

\section{MODEL OF THE GRANULES LOCATION ON THE SURFACE OF TEXTURED COATING}

Right after applying the paintwork material to the primer layer of a cold-rolled steel sheet, granules are located randomly on the surface and are not connected to each other. At a temperature of $220^{\circ} \mathrm{C}$, the paint material polymerizes rapidly. Therefore, this temperature is called as the polymerization temperature. After the polymerization, the ends of polymer molecules are adsorbed on surfaces of neighboring granules. Then, "bridge" structures (clusters) are formed. As a result, closely spaced granules are interconnected (Figure 2). This effect appears only in a specific range of concentrations of the granules. The concentration range corresponding to flocculation depends on the molecular mass of the polymer.

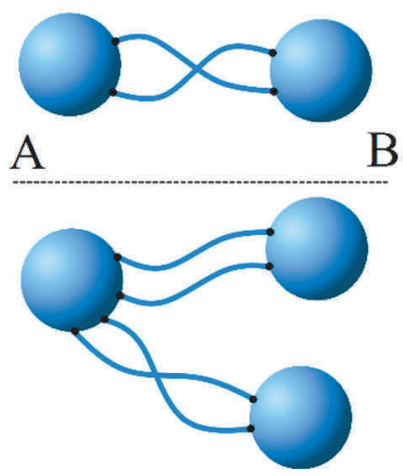

Figure 2 Flocculation scheme of two granules

For modeling the location of the granules, the DLCA (Diffusion Limited Cluster Aggregation) model is used. The number of the granules is determined. Initially, they are placed randomly on a square lattice (Figure 3a). At each step of modeling, the granules move along one of the four possible lattice directions on the distance equal to the linear size of the cell. Upon contact (being on adjacent cells), the granules merge to form a cluster. The free walking of both individual granules and clusters is considered in this simulation. At a certain stage, a single (infinite) fractal cluster remains on the lattice (Figure $3 b$ ). A dense net of associated polymer molecules is formed. Due to the infinite cluster formed, the coating becomes softer at the bending deformation. Erichsen elasticity increases in comparison with smooth polymer coatings [6]. 


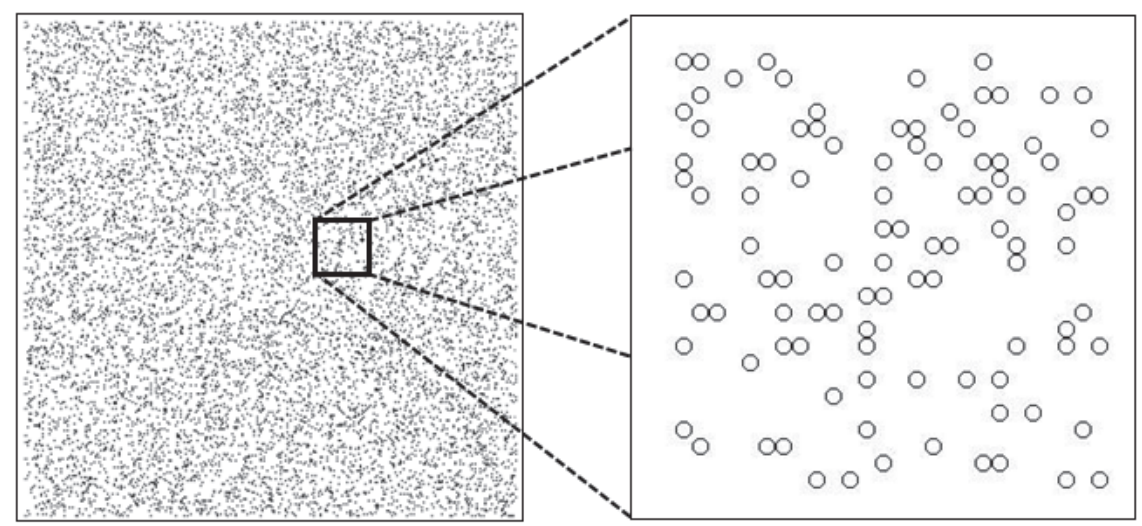

a)

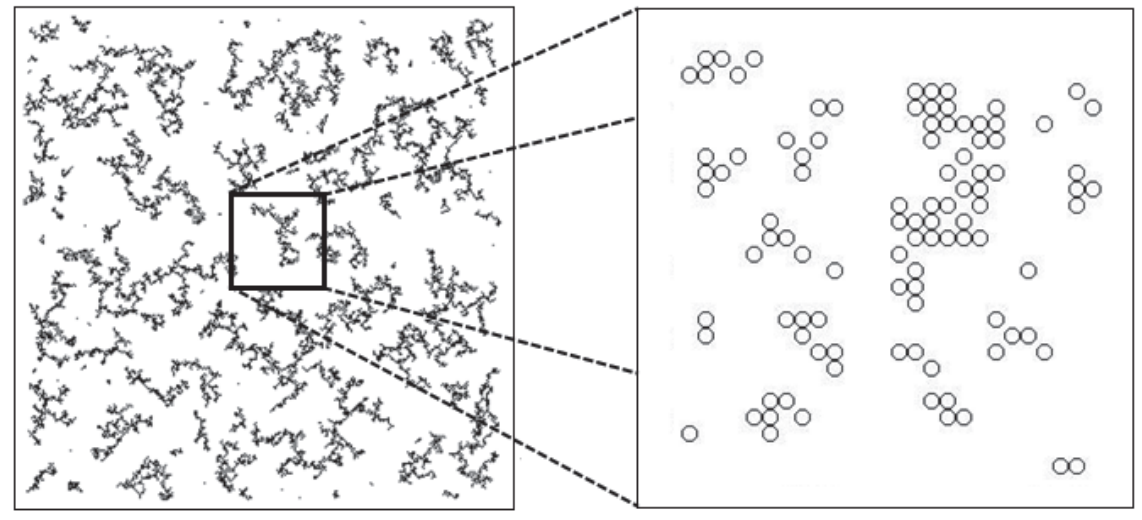

b)

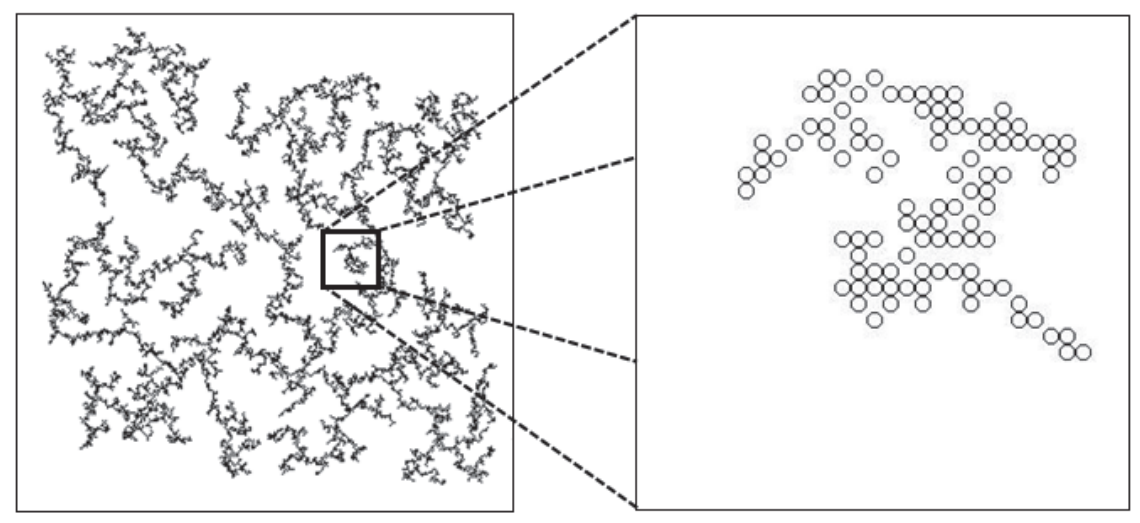

c)

Figure 3 The location of the granules on the surface of the polymer coating at time $t=0(a) ; \tau_{0} / 2(b) ; \tau_{0}$ (c). $\tau_{0}$ is the scale time of formation of an infinite cluster.

If the sample doesn't have sufficient time for the formation of the coating at the polymerization temperature, the infinite cluster is not observed (Figure 3c). In this case, lines appear (for example, the $A B$ line in Figure 2) along which the cracking of the paint is most possible when an external stress is applied. 


\section{MODEL OF THE GRANULES LOCATION OF ON THE SURFACE OF TEXTURED COATING}

To study the optical properties of textured polymer coating, it is not enough to simulate the location of the granules, since it is necessary to consider defects which sizes are comparable with the light wavelength. Therefore, the processes occurred during the formation of the coating near one granule are considered. Each granule undergoes the intensive flocculation. Some macromolecules are adsorbed in the form of loops only on the surface of one particle. To study the surface morphology near one granule, the diffusion-limited aggregation (DLA) model is used.

This model is an aggregate (cluster) growth based on random walks of particles (Brownian motion). The model proposed by T.A. Witten Jr. and L.M. Sander in 1981 proved to be widely applicable for the imitational modeling of such phenomena as the growth of mineral dendrites, bacterial colonies, the formation of a cluster by drying of colloidal solution upon glass [6].

The process of building an aggregate (cluster) begins with a granule located at the center of the considered surface area where the flocculation will take place. The initial position of the monomer on the circle surrounding the granule is randomly selected. The monomer begins to walk over the surface, according to the formula

$$
\begin{aligned}
& x_{k+1, j}=x_{k, j}+\Delta r \cdot \cos \varphi \\
& y_{k+1, j}=y_{k, j}+\Delta r \cdot \sin \varphi
\end{aligned}
$$

where:

$x_{k, j}$ and $y_{k+1, j}$ are monomer coordinates, index $j$ is its number of the step, $k$ is its number of the monomer the angle, $\varphi$ is measured from the direction of the monomer movement at the previous step

(Figure 4).

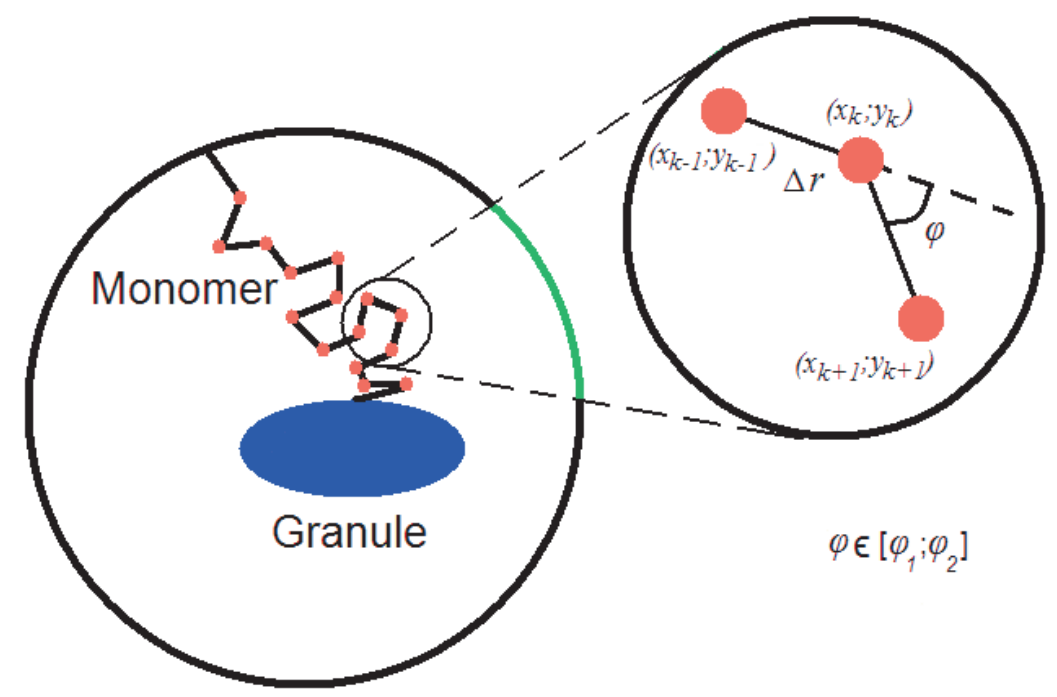

Figure 4 Diffusion-limited aggregation model (DLA)

This angle $\varphi$ takes a random value from a uniform distribution in the interval $\left[\varphi_{1} ; \varphi_{2}\right]$. The walk continues until the monomer is adjacent to any of the monomers included into the aggregate (cluster). After that, the monomer joins the cluster, becoming part of it, and then process repeats. If the monomer goes far from the cluster, then it is discarded and the next one is generated. Figure $\mathbf{5}$ shows the obtained fractal sets near the granule. Definitely, the type of surfaces obtained depends on the randomness factor. But the determining factor is the angle interval, that is, the values of the angles $\varphi_{1}$ and $\varphi_{2}$. 


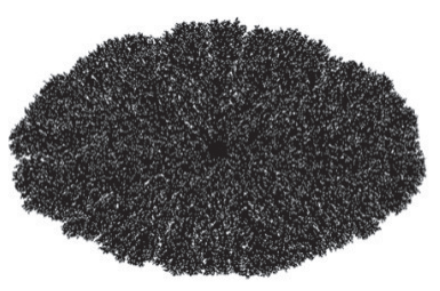

a)

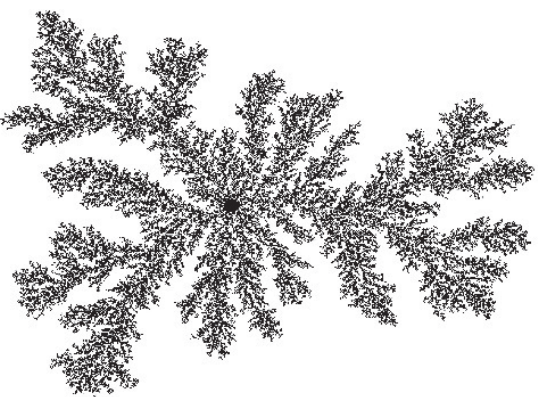

c)

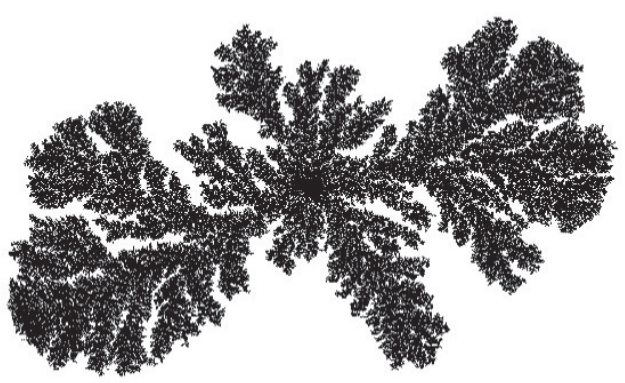

b)

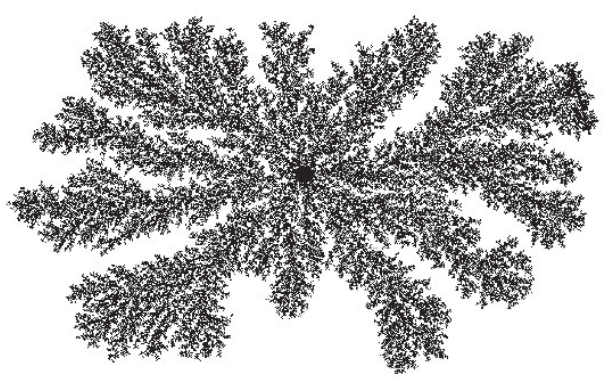

d)

Figure 5 The fractal sets obtained by the DLA method. $\varphi \in\left[-10^{\circ} ; 10^{\circ}\right]$ (a); $\varphi \in\left[-15^{\circ} ; 0^{\circ}\right]$ (b); $\varphi \in\left[0^{\circ} ; 90^{\circ}\right\rfloor(c) ; \varphi \in\left[0^{\circ} ; 360^{\circ}\right\rfloor(d)$

\section{THE RESULT OF THE SIMULATION}

As a result of combining the two models which were discussed above, 3D distribution functions (Figure 6) of defects on the surface of the textured polymer coating were constructed. It was assumed that the height of the defect is proportional to the concentration of the monomers, which are located near the granules. Comparing Figures 1 and $\mathbf{6}$, it can be concluded that the random walk method allows us to construct a surface relief of the textured metal-roll coating, which will make it possible to make a theoretical study of its optical properties. The results of industrial tests have shown that the type of defects depends on the formation conditions of the polymer coating. The theoretical appearance of the surface is determined by the magnitude of the angles $\varphi_{1}$ and $\varphi_{2}$. These parameters depend on the chemical structure of the polymer and the mode of the coating drying. The comparison results of the simulation and results obtained by the optical microscope method showed that this dependence is ambiguous. When $S=\varphi_{1}+\varphi_{2}=0$ (symmetric interval) and $\Delta \varphi=\varphi_{1}+\varphi_{2}<<360^{\circ}$, the particle moves almost straight and the fractal set has the appearance of almost solid circle (Figure 5a). It can be assumed that such a surface relief is possible in the case when flocculation occurred before polymerization, that is, the coating was formed at too low temperature and, therefore, it is defective. The fractal set corresponding to the high-quality coating (Figure 1) can be constructed with an asymmetric interval $(S \neq 0)$. We assumed that in this case the flocculation and the polymerization occur simultaneously, therefore the monomer can not move like an ordinary Brownian particle, since the movement freedom of the monomer is restricted by the macromolecule. By means of the optical microscope method, it was found that the number of beams in the clusters obtained at the higher temperature is increased. The simulation results showed that the number of beams becomes large for wider interval of $\Delta \varphi$ (Figures 5 bd). This can be explained by the fact that, the monomers have more chaotic motion and the entropy of the system increases at higher temperatures. Therefore, the value $\Delta \varphi$ needs to be increased for the modeling the morphology of the surface formed at high temperatures. 


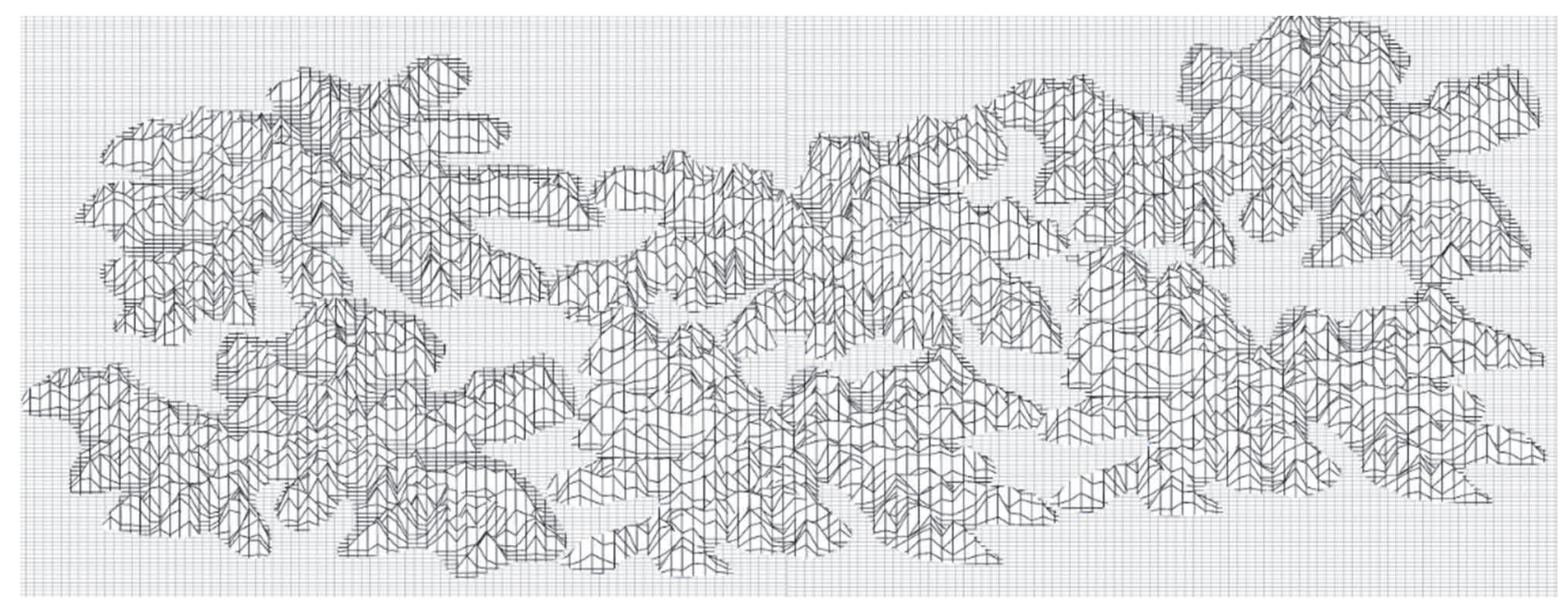

Figure 6 The surface relief of the textured metal-roll coating constructed using the random walk method

\section{CONCLUSION}

The analysis revealed that the surface of the textured polymer coating of the metal sheet can be simulated using fractal geometry. The results should be a applicable in theoretical investigations of mechanical and optical properties of the coatings.

\section{ACKNOWLEDGEMENTS}

This work was supported by the Russian Foundation of Basic Research, project № 19-42-350001.

\section{REFERENCES}

[1] ZAMANI, Morteza, SHAFIEI, Farbod, FAZELI, Seyed Mahdi, DOWNER, Michael, JAFARI, Gholam Reza. Analytic height correlation function of rough surfaces derived from light scattering. Physical Review E. 2016. vol. 94, p. 042809.

[2] SEMCHUK, Olexander, GRECHKO, Leonid, VODOPIANOV, Dmitriy, KUNITSKA, Leonid. Features of light scattering by surface fractal structures. TASK QUARTERLY. 2009. vol. 13, no. 3, pp. 199-206.

[3] MAKSIMOV, Andrei, MAKSIMOVA, Ekaterina, EGOROV, Vladislav. Modeling of light scattering by polymer coatings of rolled metal surface. IOP Conference Series: Journal of Physics. 2017. vol. 936, p. 012041.

[4] MANDELBROT, B. In: Springer Netherlands, eds. Stanley, H Eugene, Ostrowsky, N. An Random Fluctuations and Pattern Growth: Experiments and Models, 1988, Chapter 40: An Introduction to Multifractal Distribution Functions, pp. 279-291.

[5] ZHANG, Qiang, XU, Chong- Yu, YU, Zuguo, LIU, Chun-Ling, CHEN, Yongqin. Multifractal analysis of streamflow records of the East River basin (Pearl River). Physica A. 2009. vol. 388, no. 6, pp. 927-934.

[6] GOST 34180-2017. Steel cold rolled and cold rolled hot-galvanized sheet with polymer coating, prepainted by the continuous coil-coating process. Specifications.

[7] RUZHITSKAYA, Darya, RYZHIKOVA, Yuliya, RYZHIKOV, Sergei. Algorithms for analysis of the characteristics of dendritic structures. Bulletin of the Russian Academy of Sciences: Physics. 2018. vol. 82, no. 11, pp. 1512-1515. 